\title{
Asociación de HSP60 de Chlamydia trachomatis y desarrollo de cáncer de ovario
}

\author{
Association between Hsp60 of Chlamydia trachomatis and ovarian \\ cancer.
}

Adriana Paola Jutinico Shubach ${ }^{1}$ Johanna Lizeth González Devia² ${ }^{2}$ Ruth Mélida Sánchez Mora ${ }^{3}$

\section{Resumen}

Las proteínas de choque térmico (HSP) son una familia de proteínas altamente conservadas cuya expresión se regula por la exposición a condiciones de estrés. Chlamydia trachomatis (CT) es una bacteria intracelular obligada de transmisión sexual causante de infección crónica y persistente que posibilita la expresión de proteínas tipo HSP, una de estas proteínas es la HSP60 Clamidial (cHSP60) que posee una homología del 50\% con respecto a la proteína HSP60 humana. Esta revisión presenta estudios immunoepidemiológicos que han demostrado una asociación entre la respuesta inmune a cHSP60 y secuelas de la infección por CT generadas por la inflamación crónica, una de estas secuelas probablemente es el desarrollo de cáncer de ovario, patología que según la OMS es uno de los cánceres ginecológicos más letales y ocupa el quinto lugar en muertes por cáncer entre las mujeres. En esta revisión se discute como la presencia de la cHSP60 ocasiona una respuesta de anticuerpos prolongada como una fuente de estimulación antigénica e inflamación crónica, ocasionando reactividad cruzada que se asocia al desarrollo de cáncer de ovario.

\section{Palabras claves:}

\section{Abstract}

Heat Shock Proteins are a family of highly conserved proteins and their expression is regulated by exposure to stress conditions. Chlamydia trachomatis is an obligate intracellular bacterium, sexually transmitted that causes chronic and persistent infection, which enables the expression of HSP-like protein as HSP60 Chlamydial (cHSP60). cHSP60 shares 50\% homology with human protein. This review shows imunoepidemiological studies that have demonstrated an association between the immune response to cHSP60 and sequelae of CT infection generated by chronic inflammation. One of those sequelae is probably the development of ovarian cancer, a pathology that according to $\mathrm{WHO}$ is one deadliest gynaecologic cancer and the fifth leading cause of cancer dead among women. In this review, we discuss how cHSP60 causes a prolonged antibody response as a source of antigenic stimulation and chronic inflammation due to cross reactivity with human antigens and its association with the development of ovarian cancer.

\section{Keywords:}

1. Bacterióloga, Joven investigadora, Universidad Colegio Mayor de Cundinamarca.

2. Bacterióloga. Estudiante de Maestría en Bioquímica, Universidad Nacional de Colombia.

3. PhD Biotecnología. MSc Genética Humana. Docente Universidad Colegio Mayor de Cundinamarca. 


\section{Introducción}

El cáncer de ovario es la malignidad ginecológica más letal, donde su desarrollo y factores de riesgo asociados no están esclarecidos aún. Sin embargo, se conoce que la inflamación crónica, caracterizada por células inmunes activadas persistentemente, está implicada en la patogénesis de varios canceres humanos; bajo estas condiciones proteínas de choque térmico (HSP) son expresadas $(1,2)$.

Las HSP constituyen una gran familia de proteínas implicadas en el plegamiento y maduración de proteínas cuya expresión es inducida por choque térmico u otras condiciones de estrés (3). Además, se ha visto que las HSP ayudan a la protección de células epiteliales contra la apoptosis, por lo que podría mejorar el proceso de proliferación celular (4).

Por otro lado, es importante mencionar que la principal causa del $18 \%$ de los casos de canceres en el mundo, que afecta 1.2 millones de personas por ańo, es debido a agentes infecciosos (2); dentro de estos agentes, se encuentra Chlamydia trachomatis (CT), este microorganismo de transmisión sexual, genera una infección persistente que puede durar meses o ańos en el área endocervical (5).

Este estado de persistencia dentro de la célula huésped se genera a partir de la resistencia a estímulos apoptóticos ofreciendo un mecanismo patógenico asociado a inflamación crónica, que conlleva a la expresión de moléculas inmunoreactivas, tal como las HSP60 Clamidial (cHSP60), cuya homología es cercana al 50\% frente a la proteína HSP60 humana, por lo que provee una continua fuente de estimulación antigénica (6-8). Por lo anterior, se han generado nuevas hipótesis en donde existe una asociación entre la cHSP60 la cual podría favorecer el desarrollo de cáncer, como el tumor de ovario (4).

\section{Cáncer de ovario}

El cáncer de ovario, según la Organización Mundial de la Salud, es la principal causa de mortalidad por cáncer ginecológico, y es la quinta causa de muerte por cáncer más frecuente entre las mujeres $(9,10)$. En el año 2012 se reportaron 151.905 muertes relacionadas con este cáncer y tiene un impacto desproporcionado sobre la morbilidad y mortalidad del cáncer: 238.719 nuevos casos se detectaron en 2012, lo que representa un aumento en la morbilidad en comparación con 225.500 nuevos casos en $2008(9,11)$.

Aproximadamente 1900 nuevos casos de esta enfermedad son diagnosticados cada año en Colombia, donde la incidencia es de 8,7 por 10.000 mujeres y la mortalidad es de 3,1 casos por 100.000 mujeres $(9,11,12)$. Cabe mencionar, que el riesgo de contraer cáncer de ovario durante la vida de una mujer es de 1 en 75 y la oportunidad de morir por esta enfermedad es de 1 en 100 (13).

En adición, esta enfermedad es generalmente diagnosticada tardíamente, a una edad de 56 a 63 ańos en la población colombiana y en estados avanzados (etapas III y IV), ya que la mayoría de veces es asintomática o causa molestias abdominales menores (12). Existen diversos tipos de cáncer de acuerdo a su histología, sin embargo el más frecuente es el seroso papilar, seguido por las variedades mucinoso, endometrioide, células claras, transicional, mixto e indiferenciado $(12,14)$.

Su origen es en la superficie del ovario (germinal) epitelial y su progresión hacia la malignidad es acompańada por la pérdida de la diferenciación epitelial y un cambio hacia un fenotipo mesenquimal; esto se conoce como transición epitelio-mesenquimal (EMT) $(14,15)$. La EMT se caracteriza por el aumento de la expresión de marcadores mesenquimales como vimentina, trombospondina, $\mathrm{N}$-cadherina y vitronectina; mientras que hay una disminución en la expresión de marcadores epiteliales como E-cadherina, Occludina, JUP, y Mucin1. Con respecto a los carcinomas avanzados, las células mesenquimales pueden recuperar características de células epiteliales, por lo tanto se conoce como transición mesenquimo-epitelial (MET) (15). 
Por otra parte, a pesar de que esta patogénesis ha recibido muy poca consideración, se ha asociado a la enfermedad pélvica inflamatoria (EPI) y otros factores como edad, paridad, uso de anticonceptivos orales e infertilidad. Además de lo anterior, se ha postulado su desarrollo a partir de infecciones por agentes microbianos como lo es Chlamydia trachomatis, la cual es la causa más común de EPI en el mundo (16).

\section{Chlamydia trachomatis}

Chlamydia trachomatis (CT) es una bacteria intracelular obligada (17), Gram negativa, no móvil y aeróbica (18). Es un patógeno del humano perteneciente a la familia Chlamydiaceae y considerada uno de los microorganismos de transmisión sexual más prevalentes en el mundo (17). Es capaz de producir en mujeres uretritis, salpingitis, endometriosis, cervicitis, embarazo ectópico, enfermedad pélvica inflamatoria (EPI), infertilidad del factor tubular, entre otras enfermedades (19-21).

Se conoce que CT tiene una prevalencia creciente con casi 100 millones de nuevas infecciones cada ańo (22), de las cuales no son diagnosticados a tiempo, debido a que el $80 \%$ de los casos se presentan de forma asintomática, donde alrededor del $70 \%$ son mujeres y de un $30 \%$ a un $50 \%$ son hombres $(23,24)$.

Esta bacteria puede generar un estado de persistencia en el área endocervical durante un tiempo prolongado. Además, las infecciones repetidas o un tratamiento antimicrobiano tardío son factores que favorecen la invasión de órganos genitales superiores, como los ovarios o las trompas de Falopio (5).

Lo anterior puede generar enfermedad crónica inflamatoria que en investigaciones se ha mostrado como posible promotor o iniciador de cáncer. Por tal motivo, CT se ha considerado como un cofactor en cáncer cervical y asociado principalmente con células escamosas de carcinoma de cérvix (16).

\section{Relación del ciclo de vida de Chlamydia trachomatis con HSP6O}

El ciclo de vida de CT es bifásico, en su forma infecciosa, denominada Cuerpo Elemental (CE) o metabólicamente inactivo, mide $0.3 \mu$ aproximadamente y está ubicado extracelularmente. El CE se une a una superficie de la célula epitelial y se incorpora en un fagosoma o vacuola de inclusión, que migra a la región distal del aparato de Golgi. Dentro de la vacuola, la inclusión se diferencia a una forma metabólicamente activa, no infecciosa, que es denominado Cuerpo Reticulado (CR) y mide 1,0 $\mu$ aproximadamente $(25,26)$. Los CR se diferencian en nuevos $\mathrm{CE}$ y son liberados por lisis celular o exocitosis en el medio extracelular donde infectan células vecinas, como se representa en la Figura $1(25,27)$.

Por lo que respecta la duración del ciclo de CT, a nivel in vivo es complejo y su desarrollo puede ser perturbado; sin embargo, a nivel in vitro su desarrollo puede completarse entre 2 y 3 días (36). Además, ciertas circunstancias como la deficiencia de nutrientes, interferón-gamma, antibióticos, pueden resultar en alteraciones morfológicas de los CRs y la aparición de formas atípicas clamidiales (36). Estas formas aberrantes pueden persistir dentro de la célula huésped en un estado viable, sin ser detectadas por cultivo celular por largo tiempo (36), figura 1. 


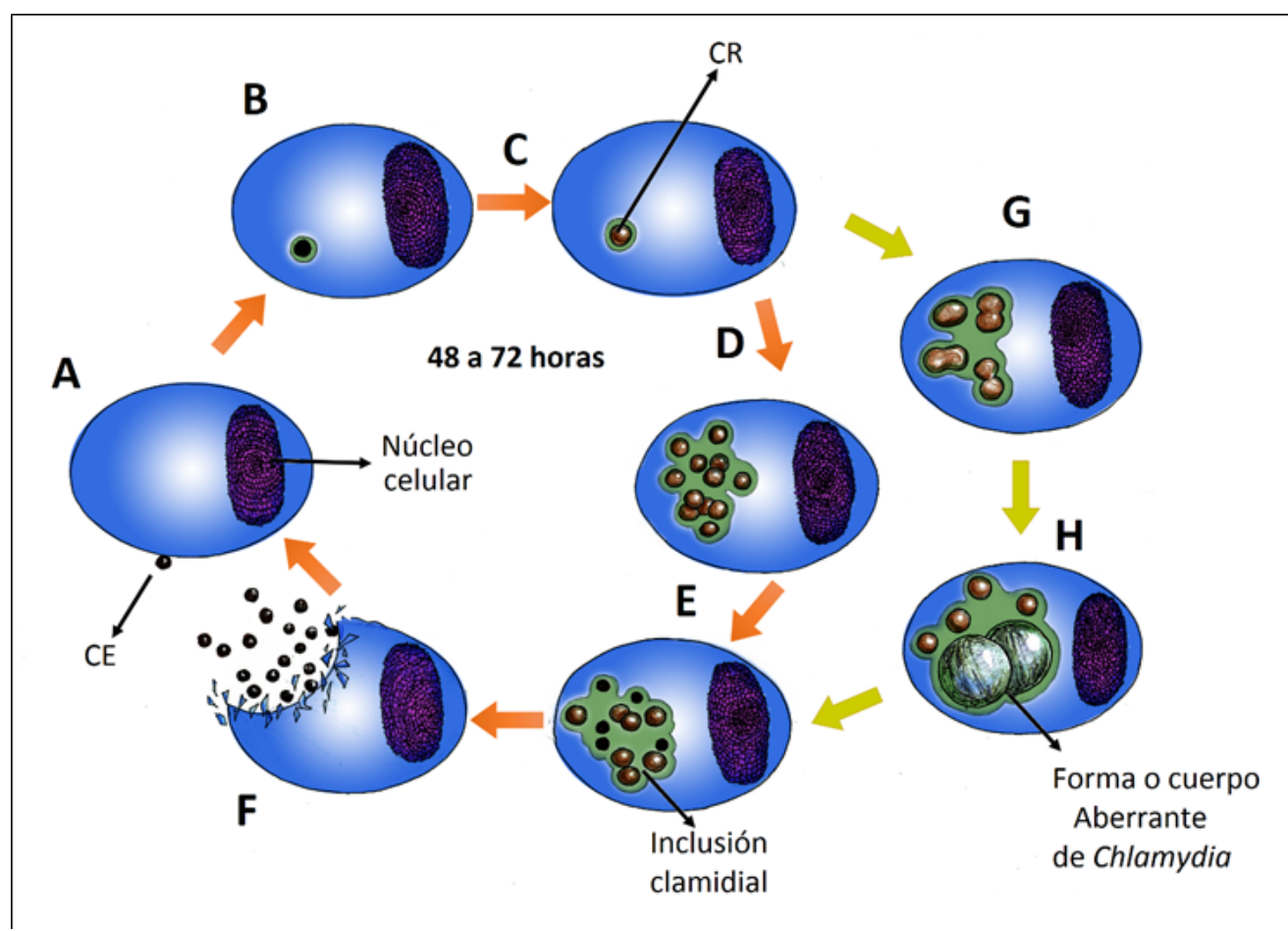

Figura 1. Ciclo de vida de Chlamydia trachomatis. El ciclo de vida de CT tiene una duración de 48 a 72 horas aproximadamente, en donde la forma infecciósa o Cuerpo Elemental (CE), se adhiere y entra a la célula del huésped rodeada de una membrana (A). Se produce una inhibición de la fusión fagolisosomal (B) que permite la diferenciación primaria de CE a Cuerpo Reticular (CR) (C). Posteriormente, los CR se dividen varias veces por fisión binaria (D) que provoca la diferenciación secundaria de CR a CE (E); y finalmente, por exocitosis o lisis celular se liberan los CE (F) para infectar células vecinas. Sin embargo, CT es capaz de persistir en el huésped gracias a la producción de interferón-Y (INF Y), que mediante la inducción de la enzima indolamina triptófano-degradantes 2,3-dioxigenasa (IDO), reduce los niveles intracelulares de triptófano y frena la replicación de CR (G). Estos CR no son infecciosos, pero permanecen viables denominándose Cuerpos Aberrantes $(\mathrm{H})$. Durante este estado, los genes que codifican para proteínas implicadas en la replicación, generación de energía, y producción de componentes estructurales de CT, son inactivados y en contraste, el gen que codifica para HSP60 se incrementa y es liberado al medio extracelular como respuesta a tensiones externas.

Fuente: Elaborada por los autores

Como se mencionó, una característica importante de CT es la capacidad para persistir en el huésped luego de estímulos inmunitarios (35). Durante la infección la respuesta inmune del huésped es activada a través de la producción de interferón- $Y$ (INFY), y citocinas proinflamatorias. Estos mediadores combaten eficazmente la infección extracelular, pero su influencia intracelular, especialmente del INFy, es compleja (35).

El INFy mediante la inducción de la enzima indolamina triptófano-degradantes 2,3-dioxigenasa (IDO) reduce los niveles intracelulares de triptófano lo que permite frenar la replicación CR (35, 37); sin embargo, los CR permanecen viables y se convierten en lo que es conocido como una forma persistente $(36,37)$, ver Figura 1. Además de lo anterior, la persistencia por CT también puede ser inducida por el factor de necrosis tumoral a (FNT a) e in vitro, mediante la adición de penicilina en los cultivos celulares de CT (35).

Durante el estado persistente, los genes que codifican para proteínas implicadas en la replicación, la generación de energía, y en la producción de componentes estructurales de CT, son inactivados (35). En marcado contraste, el gen que codifica para cHSP60 se incrementa y la proteína es liberada al medio extracelular como respuesta a tensiones externas $(35,36)$. 


\section{Proteinas de choque térmico}

Las proteínas de choque térmico (HSP) son una familia de proteínas altamente conservadas que representa entre el $1 \%$ y $2 \%$ de la reserva total de proteínas. Su expresión es regulada hasta después de la exposición a condiciones de estrés tales como; choque térmico, estrés oxidativo, inflamación, irradiación $(28,29)$. Aunque todas las funciones todavía no están totalmente esclarecidas, la mayoría de las HSP actúan como chaperonas moleculares, es decir que se ligan a los polipéptidos generados en el retículo endoplásmico y acompañan a las diferentes organelas en donde adquieren su estructura tridimensional para realizar funciones esenciales que contribuyen al buen plegamiento de las proteínas y reparación del daño de las mismas $(28,29)$.

Así mismo, se ha visto el papel de las HSP y su participación en varios procesos de carcinogénesis e incluso en la resistencia a tratamientos anticancerígenos (30). Las HSP se expresan en numerosas neoplasias malignas y participan en la proliferación del tumor, diferenciación, invasión, metástasis muerte y reconocimiento por parte del sistema inmune. Lo anterior, es debido a que durante la protección celular contra la apoptosis son inducidos factores como citoquinas, radiación ionizante o estrés oxidativo; y por otra parte, la expresión excesiva de estas proteínas induce el estrés celular crónico que resulta en la inhibición de la apoptosis y facilita la transformación a células neoplásicas (30).
Por otro lado, cabe resaltar que dentro de estas proteínas se encuentra la HSP60, la cual es de alto peso molecular y es dependiente de ATP $(28,29)$.

\section{Proteina de choque térmico de $60 \mathrm{kDa}-\mathrm{HSP6O}$}

Entre las principales proteínas de choque térmico esta la chaperona HSP60, también conocida como GroEL y/o Cpn60. Es una proteína codificada por el gen HSPD1 (posición 2q33.1), compuesta por 573 aminoácidos lo cual corresponde a un peso molecular de $61,05 \mathrm{kDa}(31)$. Hace parte de la familia de polipéptidos, cuyas secuencias aminoacídicas están altamente conservadas tanto en procariotas como en eucariotas y son producidas por microorganismos y tejidos de mamíferos después de la exposición a varias condiciones de estrés $(32,33)$.

En organismos eucariotas, esta principalmente localizada en las mitocondrias donde interactúa con cofactores como GroES o HSP10 donde actúa como chaperonina para evitar la agregación al proporcionar el plegamiento correcto de las proteínas $(28,32,34)$, ver Figura 2. Sin embargo, esta proteína también se encuentra localizada en el citosol, la superficie celular, el espacio extracelular e incluso en la sangre periférica; y se expresa en los diferentes tejidos y órganos, incluyendo el sistema inmune, el tejido epitelial y otras células $(3,32,35)$, ver Imagen 1.

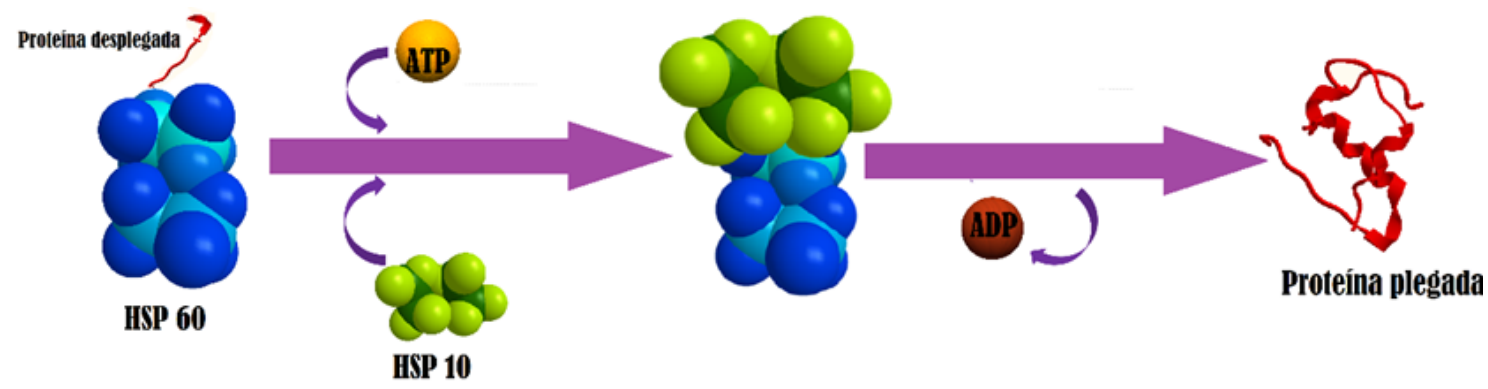

Figura 2. Plegamiento de proteínas a través de HSP60. Hsp60 trabaja en cooperación con Hsp10 para ayudar en el plegamiento de proteínas mitocondriales no plegadas a través de un mecanismo dependiente de ATP. Fuente: Elaborada por los autores 


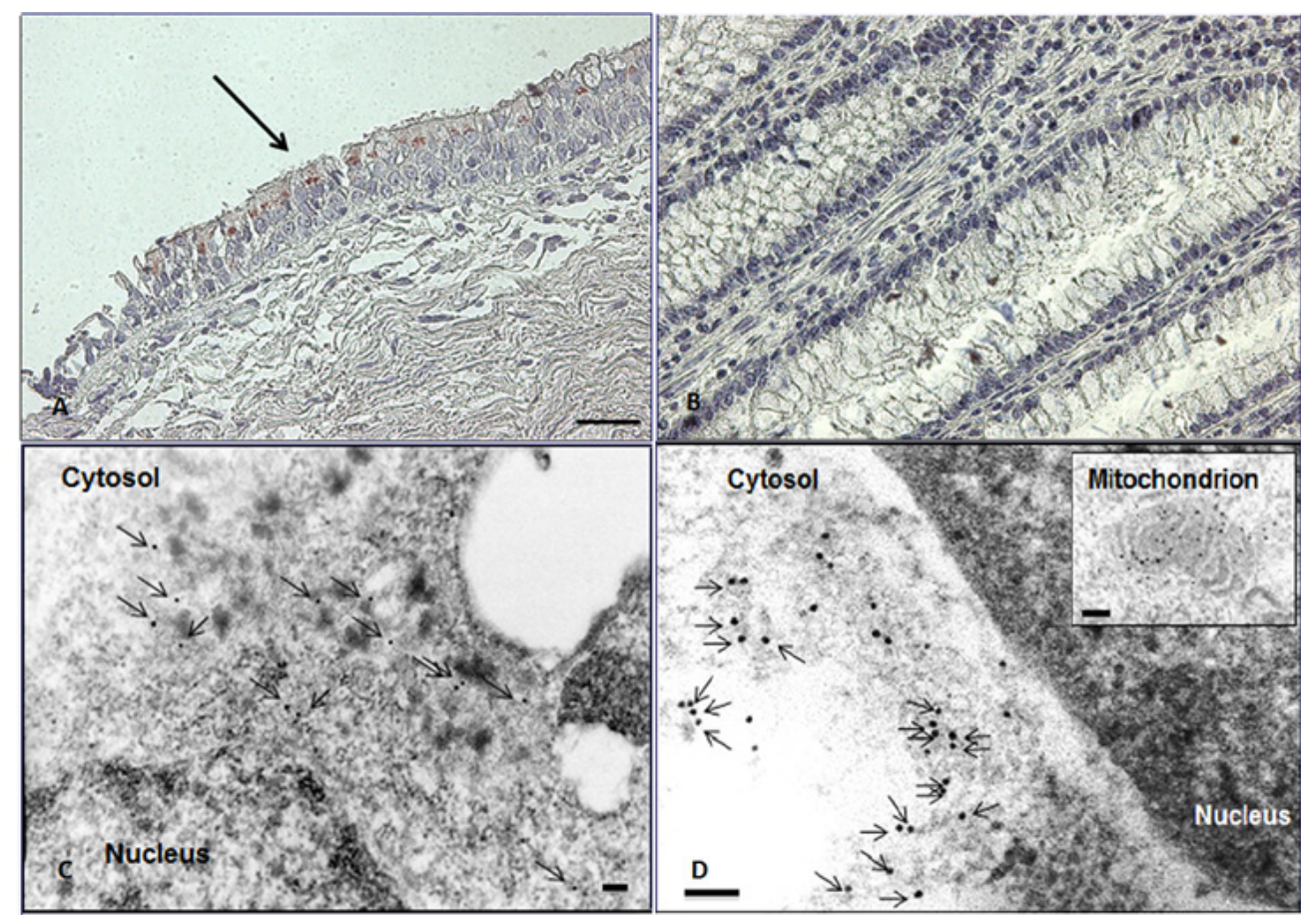

Imagen 1. Expresión y localización de HSP60 A: Mucosa bronquial humana no patológica. Las células ciliadas presentan positividad para Hsp60 por inmunohistoquímica (IHC) en el citosol (manchas rojas). B: Mucosa de colon humano no patológico. Las células intestinales no muestran positividad para Hsp60 en IHC, ya que la proteína está bajo el umbral de detectabilidad. C: Células epiteliales de mucosa de colon inflamado (paciente con colitis ulcerosa) que muestran positividad para Hsp60 (inmunoelectrónica) en el citosol (flechas). D: Células pulmonares de carcinoma mucoepidermoide que muestran positividad para Hsp60 (IHC) en el citosol y en membrana plasmática (flechas). Recuadro: Positividad mitocondrial clásica de Hsp60. Fuente: Imagen tomada y modificada de Campanella y cols, 2012; Cappello y cols, 2011; Tomasello cols, 2011 (35-37).

Por otro lado, una de las principales características son las similitudes estructurales y funcionales significativas entre varias especies de procariotas y eucariotas, además de las variaciones notables en la estructura del gen hsp60 y sus isoformas, lo que sugiere que este gen ha evolucionado con diversidad funcional (32).

\section{HSP60 Clamidial}

La familia de HSP60 es distribuida y conservada ampliamente y tiene una homología de aproximadamente $50 \%$ entre el humano y otras especies como CT (38). Esta proteína conocida como HSP60 clamidial (cHSP60) esta codificada en el locus GroEL (39). Existen tres genes codificadores de cHSP60; estos son el gen GroEL1 (Ct110), GroEL2 (Ct604) y GroEL3 (Ct755) (7,39).
Análisis transcripcionales, muestran que los tres genes GroEL clamidiales no son pseudogenes. Análisis filogenéticos y de choque térmico indican que GroEL2 y GroEL3 podrían tener funciones distintas a GroEL1; y además, son regulados de manera diferente (40).

En el año 2004, Gérard H. publicó un estudio sobre la expresión de dichos genes, tanto en infección activa como persistente, e indicó que el mRNA durante la infección activa, codifica como producto mayoritario los genes Ct110, Ct604 y Ct755; mientras que durante la infección persistente, la expresión de Ct604 predominaba sobre Ct110 y Ct755, los cuales se encontraban ausentes (39).

Por otro lado, cHSP60 se encuentra en un complejo externo de membrana de CT (7). Análisis estructu- 
rales han demostrado la presencia de los dominios de cHSP60-1, cHSP60-2, cHSP60-3 en las vesículas de inclusión de CT. Donde, cHSP60-1 está ubicada dentro de la inclusión confinado al cuerpo reticular; cHSP60-2 está ubicada en el cuerpo reticular, de forma libre dentro de la inclusión, en la superficie de las células huésped y liberado extracelularmente; y cHSP60-3, se ha visto con un patrón similar con respecto a cHSP60-2. Cabe mencionar, que la importancia de la salida de la inclusión de cHSP60-2 y cHSP60-3 frente a la cHSP60-1 se desconoce en este momento (19).

Con respecto a la expresión de esta proteína, es relevante mencionar su aumento durante infecciones persistentes, donde es reconocida como inmunoantígeno; por lo tanto, se ha encontrado una asociación entre la respuesta inmune a cHSP60 y secuelas de la infección por CT (7). Específicamente, se propone que existe una respuesta autoinmune en la cual hay producción de anticuerpos contra cHSP60, los cuales son inadvertidamente dirigidos contras las proteínas homologas HSP60 presentes en el tejido humano; y como consecuencia se inducen daños a los tejidos por un mecanismo que aún no está claro $(38,41)$.

\section{Mimetismo molecular}

La gran homología entre las HSP60 de varias especies bacterianas y la humana ha llevado a postular su participación en los fenómenos de mimetismo molecular con implicaciones patogénicas $(36,42)$, e incluso postular su papel en manifestaciones autoinmunes y/o actuar como receptores de acetilcolina (31,43-46).

Hasta ahora existen más de 150 homólogos de secuencias de HSP60 con similitud entre un 40\% y un $100 \%$ a nivel de aminoácidos. Entre estos homólogos se pueden encontrar la HSP60 de rata (Rattus norvegicus), pez globo (Fugu rubripes), pez cebra (Danio rerio), el nematodo Caenorhabditis elegans, ratón (Mus musculus) y CT $(31,47)$.
Como se ha mencionado el mimetismo molecular entre HSP60 y cHSP60 puede generar una respuesta inmune. Se ha propuesto que las adherencias y la obstrucción tubárica son resultado de una respuesta autoinmunitaria órgano específica, debido a la producción de anticuerpos contra cHSP60 (5).

Lo anterior, se ha concluido gracias a estudios que han demostrado que pacientes con oclusión tubárica y/o adherencias, muestran concentraciones elevadas de anticuerpos anti-cHSP60 debido a la alta producción del antígeno durante la infección crónica por CT. Es así, como los efectos de la reactividad cruzada contra HSP60 humanas posiblemente perpetúan y aumentan la infección; además de inducir un proceso inflamatorio con daño del tejido y posterior reparación del mismo acompañado de cicatrización y fibrosis. A partir de esto, se genera una obstrucción parcial o total de las salpinges y formación de adherencias (5).

\section{Presencia de anticuerpos anti-cHSP6O en algu- nas enfermedades}

Como se ha mencionado anteriormente, la infección persistente generada por CT favorece la expresión de proteínas genéticamente similares a proteínas humanas. Es así como cHSP60, se vuelve una proteína importante para su estudio, ya que a partir de exposiciones repetidas o reiterados puede generarse una respuesta de anticuerpos prolongada, la activación de macrófagos y una respuesta patogénica inmune, que contribuyen a promover enfermedades crónicas y daños del tejido (6).

Evidencia implica que cHSP60 como antígeno, ha mostrado el extensivo incremento de anticuerpos anti-cHSP60 y en enfermedades como EPI la titulación de anticuerpos ha sido alta (6). También se ha demostrado que el alto porcentaje de infecciones persistentes, junto con la edad, son un riesgo para el desarrollo de cáncer de ovario (16).

Por ejemplo, Ness et al en el año 2003, asoció la enfermedad pélvica inflamatoria (EPI) con el de- 
sarrollo de cáncer, ya que a partir de la medición de anticuerpos contra los tres productos del gen GroEL y contra los EB de CT serovar D en 117 mujeres con diagnóstico para cáncer de ovario; se encontró que la probabilidad de tener cáncer de ovario fue de $90 \%$ mayor cuando se tenían altos niveles de anticuerpos contra CE de CT. Además se encontró la tendencia asociada a la presencia de cHSP60-1, pero no con cHSP60-2 o cHSP60-3 (48). En contraste, Money D. et al en 1997, demostraron que no sólo se ha encontrado títulos altos de anticuerpos anti-cHSP60 en pacientes con EPI, sino también en pacientes con enfermedades como salpingitis y perihepatitis, cuyos títulos fueron significativamente altos (38).

Resultados similares se encontraron en el mismo año, donde Paavonen observó la respuesta de anticuerpos séricos a las tres proteínas de cHSP60 y su asociación con mayor riesgo de cáncer de cuello uterino. Gracias a esto encontró que los anticuerpos frente a cHSP60-1 están asociados con un mayor riesgo de carcinoma de células escamosas de cuello uterino, especialmente entre aquellos con largo tiempo de latencia, mientras que los anticuerpos a cHSP60-2 y cHSP60-3 no lo eran. Pocos casos presentaron anticuerpos contra las proteínas cHSP60 2 y 3 encontrando una asociación baja (7).

Por otro lado, Claman, P. y colaboradores en 1997 propusieron una fuerte asociación entre la respuesta de anticuerpos a la cHSP60 y embarazo ectópico como también el desarrollo de infertilidad tubárica asociada a CT. Este estudio encontró en 77 pacientes que el anticuerpo cHSP60 estaba presente en $44 \%$ de las mujeres con infertilidad por factor tubárico y sólo el $8 \%$ de las mujeres con otras causas de infertilidad. Se observó que este anticuerpo era muy predictivo para el diagnóstico de CT asociado al factor de infertilidad tubárica y se propuso como prueba de detección la microinmunofluorescencia. Aunque la sensibilidad de esta prueba para detectar la enfermedad tubárica es baja, el 92\% de especificidad de esta prueba hace que sea un ensayo importante (49).
Otras enfermedades en las que se ve implicada la presencia de anticuerpos contra cHSP60 es la enfermedad de la arteria coronaria (CAD), observado en 2002; sin embargo, anticuerpos homólogos como la HSP60 humana y de Escherichia coli, no tienen relación con esta enfermedad. Por lo anterior, se sugirió utilizar como marcador para CAD severa la respuesta de anticuerpos IgG contra cHSP60 (50).

El estudio de anticuerpos anti-cHSP60 se ha llevado a cabo junto con el análisis de anticuerpos anti-CT. En el año 2004, Cortiñas P. realizó un estudio para establecer la relación entre la presencia de dichos anticuerpos tanto en suero como en fluido folicular, en mujeres infértiles cuya causa era enfermedad tubárica, endometriosis, esterilización tubárica y/o no ovulación. De lo anterior, se detectó IgG anti-CT en el 56\% del suero e IgA anti-CT en el 37\% de los fluidos foliculares de las pacientes que tuvieron fallas en la fertilización in vitro. La presencia de anticuerpos anti-CT y anti-HSP60 podría estar relacionada con la insuficiencia de infertilización in vitro por bloqueo del desarrollo del embrión. Estos resultados destacan la importancia de diagnóstico precoz y el tratamiento de la infección por CT para evitar secuelas en el tracto genital (41).

Por último, resultados de estudios se suman con fuerza al creciente cuerpo de evidencia, que sugiere que la inmunidad anti-CT específica y anti-cHSP60 se asocia con el fracaso del embarazo, daño tisular de las trompas de Falopio, embarazo ectópico y desarrollo de tumor de ovario; ya que en el 2012, Daponte y colaboradores encontraron que las respuestas humorales dirigidas a antígenos de CT, y en particular a la cHSP60, son más prevalentes en el embarazo ectópico y en mujeres con embarazos fallidos (51).

\section{Asociación entre cHSP60 y cáncer de ovario}

Hasta aquí se ha mencionado la infección persistente de CT como factor de riesgo para el cáncer de ovario. Autores como Carvalho et al, proponen que los carcinomas de ovario serosos, son neopla- 
sias de las fimbrias tubáricas, que resultan de la infección con CT, lo cual es soportado bajo la evidencia de que los carcinomas serosos de alto grado son originados en la fimbria, existe una fuerte relación entre inflamación crónica y carcinogénesis; y CT es uno de los agentes más importantes que causa inflamación tubal crónica que puede ser relacionado con carcinogénesis (52).

Durante la infección persistente, la respuesta inmune iniciada por la infección puede resultar en una reactividad cruzada con células y o tejidos humanos por el mimetismo molecular de cHSP60 (53); esto genera el desarrollo del fenómeno inflamatorio y probablemente un proceso de autoinmunidad órgano específico con una respuesta de anticuerpos prolongada e inflamación crónica que generan en las células afectadas por lo que es considerado como promotor de cáncer $(8,16,54)$.

Además, se ha demostrado que la proteína HSP60 es reconocida como antígeno por los linfocitos $\mathrm{B}$ y T, además de ser un ligando para los receptores tipo Toll (3). En el 2006, se demostró que HSP60 induce la liberación de mediadores proinflamatorios, tal como el factor de necrosis tumoral, óxido nítrico, interleucina (IL) -6, a partir de las células del sistema inmune innato tales como macrófagos y células dendríticas; además se ha encontrado que estimula la producción de células T ayudadoras 1 e interleucinas IL-12 y IL-15 (28).

En el 2010, se realizó la identificación de antígenos Chlamydiales, a través del uso de análisis proteómicas de dos dimensiones en suero de pacientes con infecciones invasivas de CT del tracto genital superior femenino. Encontraron que la frecuencia de HSP60 fue de 100\%, es decir que se presentaba en las 13 muestras estudiadas. Otros inmunógenos de CT detectados fueron cHSP70, MOMP y PmpD (55).

Para explorar la asociación, en el año 2009 se evaluó la presencia de anticuerpos IgG contra CT e IgG anti cHSP60; donde se encontró titulaciones generalmente altas de IgG cHSP60-1 en el plasma de mujeres sometidas a cirugía debido a sospecha de cáncer de ovario; en cuanto a los anticuerpos IgG CT no fueron significativamente altos para realizar la asociación con tumor de ovario. También se encontró que la prevalencia de IgG HSP60-c, posterior a un ańo de diagnóstico fue de $86 \%$. En adición se estudió la presencia de CT en 312 muestras de tejido ovárico, de las cuales ninguna fue positiva (16).

La infección por CT puede estar implicada en la inflamación crónica de trompas y posterior carcinogénesis fimbrial. Precursores intraepiteliales fimbriales pueden evolucionar en alto grado seroso a carcinomas que se propagan rápidamente a la superficie del ovario y el peritoneo; estos tumores pueden aparecer como neoplasias primarias ováricas, aunque en realidad pueden ser una malignidad secundaria. Esta hipótesis debe investigarse más a fondo para entender las vías intracelulares de señalización implicadas en la infección por CT y su relación con carcinogénesis con el fin de descubrir posibles dianas moleculares terapéuticos (54).

Otra hipótesis planteada, sugiere que algunos tipos de cáncer de ovario, probablemente los más agresivos, pueden no tener un origen en el ovario mismo, sino que pueden surgir en las trompas uterinas. La inflamación del tubo uterino, como se mencionó anteriormente puede ser originada por la infección por CT, por tal motivo se postula en la inflamación crónica de trompas y posterior carcinogénesis fimbrial. Precursores intraepitelial fimbriales pueden evolucionar en alto grado seroso a carcinomas que se propagan rápidamente a la superficie del ovario y el peritoneo; estos tumores pueden aparecer como neoplasias ováricas primarias, o pueden en realidad ser una malignidad secundaria (52).

Es así como finalmente se resalta que la activación del sistema inmune es un factor importante que promueve el dańo del tejido. Estos sistemas podrían ser activados por la presencia de inmunógenos (cHSP60) que son expresados durante la 
infección persistente de CT $(33,38,55-58)$. El uso de estudios que detectan titulaciones altas de anti-cHSP60 en pacientes diagnosticadas con cáncer de ovario es un soporte que fortalece las hipótesis como posible factor de riesgo; sin embargo, es imprescindible continuar con el estudio de la relación de CT y tumor de ovario, en la cual anticuerpos anti-cHSP60 podrían ser usados como marcador para la detección de dicha enfermedad.

\section{Referencias}

1. 1. Carvalho JP. Is Chlamydia-infected tubal fimbria the origin of ovarian cancer? Med Hypotheses [Internet]. Elsevier Ltd; 2008;71(5):690-3. Disponible en: http://dx.doi.org/10.1016/j.mehy.2008.06.028

2. 2. Westbrook AM, Szakmary A, Schiestl RH. Mechanisms of intestinal inflammation and development of associated cancers: Lessons learned from mouse models. Mutat Res Mutat Res [Internet]. 2010 [cited 2017 Jul 19];705(1):40-59. Disponible en: http://www.sciencedirect.com.ezproxy.unal.edu. co/science/article/pii/S1383574210000244

3. 3. Wu J, Liu T, Rios Z, Mei Q, Lin X, Cao S. Heat Shock Proteins and Cancer. Trends Pharmacol Sci [Internet]. 2017 [cited 2017 Jul 19];38(3):226-56. Disponible en: http:// www.sciencedirect.com.ezproxy.unal.edu.co/science/article/ pii/S0165614716301675

4. 4. Alshamsan A, Khan S, Imran A, Aljuffali IA, Alsaleh K. Prediction of Chlamydia pneumoniae protein localization in host mitochondria and cytoplasm and possible involvements in lung cancer etiology: a computational approach. Saudi Pharm J [Internet]. 2017 May [cited 2017 Jul 19]; Disponible en: http://linkinghub.elsevier.com/retrieve/pii/ S1319016417301226

5. 5. Villagrana J, López M, Flores V, Haro MJ De, Escobedo M, Guerra F. Persistencia de Chlamydia trachomatis en el endometrio y líquido peritoneal de pacientes con infertilidad pero cultivo cervical negativo. Ginecol Obs MEx. $2013 ; 81: 23-8$

6. 6. Debattista J. Immunopathogenesis of chlamydia trachomatis infections in women. Fertil Steril. 2003 Jun;79(6):127387.

7. 7. Paavonen J, Karunakaran KP, Noguchi Y, Anttila T, Bloigu A, Dillner J, et al. Serum antibody response to the heat shock protein 60 of Chlamydia trachomatis in women with developing cervical cancer囚. Am J Obstet Gynecol. 2003 Nov;189(5):1287-92.

8. 8. Paavonen J. Sexually transmitted chlamydial infections and subfertility. 2004;1266:277-86.

9. 9. OMS. Epithelial ovarian cancer. Union for International
Cancer Control. 2014.

10. 10. Güth U, Arndt V, Stadlmann S, Huang DJ, Singer G. Epidemiology in ovarian carcinoma: Lessons from autopsy. Gynecol Oncol. Elsevier Inc.; 2015;138(2):417-20.

11. 11. Rodríguez AO, Llacuachaqui M, Pardo GG, Royer R, Larson G, Weitzel JN, et al. BRCA1 and BRCA2 mutations among ovarian cancer patients from Colombia. Gynecol Oncol. Elsevier Inc.; 2012;124(2):236-43.

12. 12. González M, Barrera CA, Castillo JS. Cáncer epitelial avanzado de ovario en un grupo de pacientes colombianas. Rev Colomb Cancerol. 2014;17(4):142-8.

13. 13. American Cancer Society. What are the key statistics about ovarian cancer? 2015.

14. 14. Janson P, Sundfeldt K, Piontkewitz Y, Ivarsson K, Nilsson $\mathrm{O}$, Enerback S, et al. E-Cadherin Expression in Human Epithelial Ovarian Cancer and Normal Ovary. 1997;280(February):275-80.

15. 15. Strauss R, Li ZY, Liu Y, Beyer I, Persson J, Sova P, et al. Analysis of epithelial and mesenchymal markers in ovarian cancer reveals phenotypic heterogeneity and plasticity. PLoS One. 2011;6(1):1-20.

16. 16. Idahl A. Chlamydia trachomatis as a risk factor for infertility in women and men, and ovarian tumor development. 2009.

17. 17. Hotez PJ, Bottazzi ME, Franco-Paredes C, Ault SK, Periago MR. The neglected tropical diseases of Latin America and the Caribbean: a review of disease burden and distribution and a roadmap for control and elimination. PLoS Negl Trop Dis. 2008 Jan;2(9):e300.

18. 18. Cervantes E. Monografía: Infecciones causadas por Chlamydia trachomatis. medigraphic. 2009;52(1):18-22.

19. 19. Giles DK, Whittimore JD, LaRue RW, Raulston JE, Wyrick PB. Ultrastructural analysis of chlamydial antigen-containing vesicles everting from the Chlamydia trachomatis inclusion. Microbes Infect. 2006 May;8(6):1579-91.

20. 20. Idahl A, Lundin E, Jurstrand M, Kumlin U, Elgh F, Ohlson $\mathrm{N}$, et al. Chlamydia trachomatis and Mycoplasma genitalium Plasma Antibodies in Relation to Epithelial Ovarian Tumors. 2011;2011.

21. 21. Balakrishnan A, Wang L, Li X, Ohman-Strickland P, Malatesta P, Fan H. Inhibition of chlamydial infection in the genital tract of female mice by topical application of a peptide deformylase inhibitor. Microbiol Res. 2009 Jan;164(3):33846.

22. 22. de Waaij DJ, Dubbink JH, Peters RPH, Ouburg S, Morré $S$ a. Comparison of GMT presto assay and Roche cobas ${ }^{\oplus}$ 4800 CT/NG assay for detection of Chlamydia trachomatis and Neisseria gonorrhoeae in dry swabs. J Microbiol Methods. Elsevier B.V.; 2015;118:70-4.

23. 23. Schautteet K, De Clercq E, Jönsson Y, Lagae S, Chiers $\mathrm{K}$, Cox E, et al. Protection of pigs against genital Chlamydia trachomatis challenge by parenteral or mucosal DNA immunization. Vaccine. Elsevier Ltd; 2012 Apr;30(18):2869-81. 
24. 24. Broadbent A, Horner P, Wills G, Ling A, Carzaniga R, McClure M. HIV-1 does not significantly influence Chlamydia trachomatis serovar L2 replication in vitro. Microbes Infect. Elsevier Masson SAS; 2011 Jun;13(6):575-84.

25. 25. Linhares IM, Witkin SS. Immunopathogenic consequences of Chlamydia trachomatis $60 \mathrm{kDa}$ heat shock protein expression in the female reproductive tract. 2010;467-73.

26. 26. Sävykoski T. Chlamydia pneumoniae infection, inflammation and heat shock protein 60 immunity in asthma and coronary heart disease [Internet]. Oulun yliopisto; 2003 [cited 2017 Jul 22]. Disponible en: http://jultika.oulu.fi/Record/ isbn951-42-6985-3

27. 27. Charley M. Chlamydial Life Cycle And Persistence - Chlamydia Pneumoniae [Internet]. [cited 2017 Jul 22]. Disponible en: https:/www.clicktocurecancer.info/chlamydia-pneumoniae/chlamydial-life-cycle-and-persistence.html

28. 28. Habich C, Kempe K, Gomez FJ, Lillicrap M, Gaston H, Zee R Van Der, et al. Heat shock protein 60 : Identification of specific epitopes for binding to primary macrophages. 2006;580:115-20.

29. 29. Bozaykut P, Ozer NK, Karademir B. Regulation of protein turnover by heat shock proteins. Free Radic Biol Med. Elsevier; 2014 Dec;77:195-209.

30. 30. Bodzek P, Partyka R, Damasiewicz-Bodzek A. Antibodies against Hsp60 and Hsp65 in the sera of women with ovarian cancer. J Ovarian Res. 2014;7(1):30.

31. 31. Faried A, Faried L, Capello F, Conway de Macario E, Macario AJ. HSPD1 (Heat Shock 60kDa Protein 1) [Internet]. Atlas of Genetics and Cytogenetics in Oncology and Haematology. 2014 [cited 2017 Jul 20]. Disponible en: http:// atlasgeneticsoncology.org//Genes/HSPD1ID40888ch2q33. html

32. 32. Kumar Singh M, Janardhan Reddy PV, Sreedhar a. S, Tiwari PK. Molecular characterization and expression analysis of hsp60 gene homologue of sheep blowfly, Lucilia cuprina. J Therm Biol. Elsevier; 2015;52:24-37.

33. 33. Magro CM, Crowson AN, Peeling R. Vasculitis as the Basis of Cutaneous Lesions in Reiter â€TM s Disease. 60(2).

34. 34. Ricci C, Ortore MG, Vilasi S, Carrotta R, Mangione MR, Bulone D, et al. Stability and disassembly properties of human naïve Hsp60 and bacterial GroEL chaperonins. Biophys Chem. Elsevier B.V.; 2016;208:68-75.

35. 35. Cappello F, De MacArio EC, Zummo G, MacArio AJL. Immunohistochemistry of human Hsp60 in health and disease: From autoimmunity to cancer. Methods Mol Biol [Internet]. 2011 [cited 2017 Jul 20];787:245-54. Disponible en: http://www.ncbi.nlm.nih.gov/pubmed/21898240

36. 36. Campanella C, Bucchieri F, Merendino AM, Fucarino A, Burgio G, Corona DF V., et al. The Odyssey of Hsp60 from Tumor Cells to Other Destinations Includes Plasma Membrane-Associated Stages and Golgi and Exosomal Protein-Trafficking Modalities. Metze K, editor. PLoS One [Internet]. Public Library of Science; 2012 Jul 25 [cited 2017 Jul 22];7(7):e42008. Disponible en: http://dx.plos.org/10.1371/ journal.pone.0042008

37. 37. Tomasello G, Rodolico V, Zerilli M, Martorana A, Bucchieri F, Pitruzzella A, et al. Changes in Immunohistochemical Levels and Subcellular Localization After Therapy and Correlation and Colocalization With CD68 Suggest a Pathogenetic Role of Hsp60 in Ulcerative Colitis. Appl Immunohistochem Mol Morphol [Internet]. 2011 Dec [cited 2017 Jul 29];19(6):552-61. Disponible en: http://www.ncbi.nlm.nih. gov/pubmed/21441812

38. 38. Money DM, Hawes SE, Eschenbach D a., Peeling RW, Brunham R, Wölner-Hanssen P, et al. Antibodies to the chlamydial $60 \mathrm{kd}$ heat-shock protein are associated with laparoscopically confirmed perihepatitis. Am J Obstet Gynecol. 1997 Apr;176(4):870-7.

39. 39. Ge C, Whittum-hudson JA, Schumacher HR, Hudson AP. Differential expression of three Chlamydia trachomatis hsp60-encoding genes in active vs . persistent infections. 2004;36:35-9.

40. 40. Karunakaran KP, Noguchi Y, Read TD, Cherkasov a., Kwee J, Shen C, et al. Molecular Analysis of the Multiple GroEL Proteins of Chlamydiae. J Bacteriol. 2003 Mar;185(6):1958-66.

41. 41. Cortiñas P, Muñoz G, Loureiro C, Pujol F. Follicular Fluid Antibodies to Chlamydia trachomatis and $\mathrm{Hu}-$ man Heat Shock Protein-60 kDa and Infertility in Women. 2004;35:121-5.

42. 42. Cappello F, Caramori G, Campanella C, Vicari C, Gnemmi I, Zanini A, et al. Convergent Sets of Data from In Vivo and In Vitro Methods Point to an Active Role of Hsp60 in Chronic Obstructive Pulmonary Disease Pathogenesis. Chu HW, editor. PLoS One [Internet]. 2011 Nov 28 [cited 2017 Jul 22];6(11):e28200. Disponible en: http://www.ncbi.nlm. nih.gov/pubmed/22140545

43. 43. Cappello F, Marino Gammazza A, Zummo L, Conway de Macario E, Macario AJL. Hsp60 and AChR cross-reactivity in myasthenia gravis: An update. J Neurol Sci [Internet]. 2010 May 15 [cited 2017 Jul 22];292(1-2):117-8. Disponible en: http://www.ncbi.nlm.nih.gov/pubmed/20236663

44. 44. Marino Gammazza A, Bucchieri F, Grimaldi LME, Benigno A, Conway de Macario E, Macario AJL, et al. The Molecular Anatomy of Human Hsp60 and its Similarity with that of Bacterial Orthologs and Acetylcholine Receptor Reveal a Potential Pathogenetic Role of Anti-Chaperonin Immunity in Myasthenia Gravis. Cell Mol Neurobiol [Internet]. 2012 Aug 19 [cited 2017 Jul 22];32(6):943-7. Disponible en: http:// www.ncbi.nlm.nih.gov/pubmed/22258649

45. 45. Marino Gammazza A, Rizzo M, Citarrella R, Rappa F, Campanella C, Bucchieri F, et al. Elevated blood Hsp60, its structural similarities and cross-reactivity with thyroid molecules, and its presence on the plasma membrane of oncocytes point to the chaperonin as an immunopathogenic factor in Hashimoto's thyroiditis. Cell Stress Chaperones [Internet]. 2014 May 22 [cited 2017 Jul 22];19(3):343-53. Disponible en: http://www.ncbi.nlm.nih.gov/pubmed/24057177

46. 46. Jones DB, Coulson AFW, Duff GW. Sequence homolo- 
gies between hsp60 and autoantigens. Immunol Today [Internet]. 1993 Jan [cited 2017 Jul 22];14(3):115-8. Disponible en: http://www.ncbi.nlm.nih.gov/pubmed/8466626

47. 47. Ngan F, Gupta M, Luke S. Methods for Detecting Genetic Abnormalities in Ovarian Carcinoma Using Fluorescence in situ Hybridization and Immunohistochemistry. 2006;

48. 48. Ness RB, Goodman MT, Shen C, Brunham RC. Serologic Evidence of Past Infection in Relation to Ovarian Cancer. 2003; 187:1147-52.

49. 49. Claman P, Honey L, Peeling RW, Jessamine P, Toye B. The presence of serum antibody to the chlamydial heat shock protein (CHSP60) as a diagnostic test for tubal factor infertility. Fertil Steril. 1997 Mar;67(3):501-4.

50. 50. Liuba P, Pesonen E, Paakkari I, Forelid A, Sandstrom S. ABSTRACTS - Hypertension, Vascular Disease, and Prevention 277 A Chlamydia Pneumoniae Resulted In Plaque Pormation Acute Chlamydia Pneumoniae Infection Causes Diffuse Adenosine Deaminase Inhibition Attenuates the. :60.

51. 51. Daponte A, Pournaras S, Deligeoroglou E, Skentou H, Messinis IE. Serum interleukin-1区, interleukin-8 and anti-heat shock 60 Chlamydia trachomatis antibodies as markers of ectopic pregnancy. J Reprod Immunol. Elsevier Ireland Ltd; 2012 Mar;93(2):102-8.

52. 52. Carvalho JP, Carvalho FM. Is Chlamydia-infected tubal fimbria the origin of ovarian cancer? Med Hypotheses. Elsevier Ltd; 2008 Nov;71(5):690-3.

53. 53. Hjelholt A, Christiansen G, Johannesson TG, Ingerslev $\mathrm{HJ}$, Birkelund S. Tubal factor infertility is associated with antibodies against Chlamydia trachomatis heat shock protein 60 (HSP60) but not human HSP60. Hum Reprod [Internet]. 2011 Aug 1 [cited 2017 Jul 22];26(8):2069-76. Disponible en: https://academic.oup.com/humrep/article-lookup/ doi/10.1093/humrep/der167

54. 54. López-hurtado M, Guerra-infante FM. P apel de los anticuerpos en el desarrollo de la infección por Chlamydia trachomatis y su utilidad en el diagnóstico. 2002;16(3):140-50.

55. 55. Forsbach-Birk V, Simnacher U, Pfrepper K-I, Soutschek E, Kiselev a O, Lampe MF, et al. Identification and evaluation of a combination of chlamydial antigens to support the diagnosis of severe and invasive Chlamydia trachomatis infections. Clin Microbiol Infect. European Society of Clinical Infectious Diseases; 2010 Aug;16(8):1237-44.

56. 56. Rodríguez Rojas Fredy Alberto, Barreto Ordoñez Paula Steffany, Sánchez Mora Ruth Mélida. Detección de Chlamydia trachomatis en hombres que tienen sexo con hombres en Bogotá: un estudio piloto. Nova [Internet]. 2016 Dec [cited 2017 Aug 02] ; 14( 26 ): 17-27. Available from: http://www.scielo.org.co/scielo.php?script=sci_arttext\&pi$\mathrm{d}=$ S1794-24702016000200002\&lng=en.

57. 57. Mantilla Alejandra, Martínez Jhonathan, Ramírez Mateo Santiago, Olave Luis Felipe, Jutinico Shubach Adriana Paola, Gómez María C et al . Determinación de IgG contra Chlamidya trachomatis en mujeres con artritis de la Ciudad de Bogotá D.C. Un estudio piloto. Nova [Internet]. 2016 June [cited 2017 Aug 02] ; 14( 25 ): 27-34. Available from: http://www.scielo.org.co/scielo.php?script=sci_arttext\&pi$\mathrm{d}=$ S1794-24702016000100003\&lng=en.

58. Páez, L. C. C., et al. Comparación del cultivo celular de HeLa y HEp-2: Perspectivas de estudios con Chlamydia trachomatis. 2015; Nova 13(23). 